\title{
Effet résiduel de la fertilisation minérale et du stade phénologique sur la croissance et la biomasse de Paspalum plicatulum
}

\author{
Camara SAWA ${ }^{1,2}$, Fernand TENDONKENG ${ }^{2 *}$, Emile MIEGOUE $^{2}$, Famoï BEAVOGUI $^{1}$, \\ Makan KOUROUMA ${ }^{1}$ et Etienne PAMO TEDONKENG ${ }^{2}$ \\ ${ }^{1}$ Institut de Recherche Agronomique de Guinée Conakry. BP 1523. \\ ${ }^{2}$ Laboratoire de Production et de Nutrition Animales, Département des Productions Animales, Faculté \\ d'Agronomie et des Sciences Agricoles (FASA), Université de Dschang. B P 222 Dschang, Cameroun. \\ *Auteur correspondant; E-mail :f.tendonkeng@ univ-dschang.org; Tél : (+237 696804671$)$.
}

\section{RESUME}

Le déficit fourrager corrélé au faible niveau de fertilité des sols constitue un obstacle de taille au développement des productions animales au Fouta-Djalon. La fertilisation azotée peut contribuer à l'intensification fourragère dans cette région de la Guinée. Ce travail visait à améliorer la valeur nutritionnelle des fourrages produits. Ainsi, un dispositif factoriel comparant six doses d'engrais (0, 50, 100, 150, 200 et 250 $\mathrm{kg} \mathrm{d} \mathrm{N} / \mathrm{ha}$ ) et trois stades phénologiques a été utilisé. A chaque stade phénologique, 64 plantes ont été prélevées pour les mesures de la croissance. La biomasse a été évaluée par niveau de fertilisation et par stade phénologique. Les résultats ont montré que la fertilisation et le stade phénologique influencent $(\mathrm{p}<0,05)$ la taille et le diamètre de $P$. plicatulum. Indépendamment du stade phénologique, une augmentation de la taille et du diamètre a été observée chez les plantes produites dans les parcelles fertilisées. La plus forte croissance des plantes a été observée à $200 \mathrm{~kg} \mathrm{~N} / \mathrm{ha}(79,51 ; 104,20$ et 111,69 cm respectivement à la montaison, à la floraison et après grenaison). Cependant, la biomasse la plus élevée de la plante entière, des feuilles et des tiges a été obtenue à $250 \mathrm{~kg} \mathrm{~N} / \mathrm{ha}$. En somme, la biomasse de la plante a augmenté (p < 0,05) avec le stade phénologique. A niveau résiduel de fertilisation azotée égal, la meilleure biomasse de la graminée a été induite à la floraison. Aucune différence $(\mathrm{p}>0,05)$ n'a été observée entre la biomasse produite à la floraison et après grenaison. (c) 2018 International Formulae Group. All rights reserved.

Mots clés: Arrière effet, NPK (17-17-17), phases de développement, production, Paspalum plicatulum.

\section{Residual effect of mineral fertilization and phenological stage on the growth and the biomass of Paspalum plicatulum.}

\begin{abstract}
The fodder deficit correlated with the low level of soil fertility constitutes a major obstacle to the development of animal production in Fouta-Djalon. Nitrogen fertilization can contribute to forage intensification in this region of Guinea. This work aimed at improvement the nutritional value of the fodder produced. Thus, a factorial device comparing six fertilizer doses $(0,50,100,150,200$ and $250 \mathrm{~kg} \mathrm{~N} / \mathrm{ha})$ and three phenological stages were used. At each phenological stage, 64 plants were taken for growth measurements. Biomass was assessed by level of fertilization and phenological stage. The results showed that
\end{abstract}


fertilization and phenological stage influence $(\mathrm{p}<0.05)$ the size and diameter of $P$. plicatulum. Regardless of the phenological stage, an increase in height and diameter were observed in the plants produced in the fertilized plots. The highest growth of plants were observed at $200 \mathrm{~kg} \mathrm{~N} / \mathrm{ha}(79.51,104.20$ and $111.69 \mathrm{~cm}$ respectively at bolting, flowering and after seedling). However, the highest biomass of the whole plant, leaves and stems was obtained at $250 \mathrm{~kg} \mathrm{~N} / \mathrm{ha}$. In sum, the biomass of the plant increased ( $<0.05)$ with the phenological stage. At a residual level of equal nitrogen fertilization, the best grass biomass was induced at flowering. No difference ( $p$ $>0.05$ ) was observed between the biomass produced at flowering and after seedling.

(C) 2018 International Formulae Group. All rights reserved.

Keywords: Rear effect, NPK (17-17-17), development phases, production, Paspalum plicatulum.

\section{INTRODUCTION}

La nécessité de nourrir une population sans cesse croissante dans les pays en voie de développement pousse de plus en plus les agriculteurs à étendre et à mettre en défens leurs surfaces cultivées au détriment des espaces pastoraux, ce qui a pour conséquence l'exacerbation des conflits pour l'utilisation de l'espace rural entre agriculteurs et éleveurs (Pamo et al., 2006; Fogang et al., 2013; Obulbiga et al., 2015).

En effet, cette situation conduit au rétrécissement des parcours naturels (Pamo et al., 2007), à leur déplacement vers les zones marginales et aux difficultés de satisfaire les besoins nutritionnels des animaux (Pamo et al., 2008 ; Tendonkeng et al., 2015). Une amélioration de la productivité animale peut passer non seulement par la maîtrise des systèmes de production, mais également par l'introduction et l'intensification de la production des espèces fourragères à haut rendement.

Parmi les nombreuses essences fourragères introduites au Fouta-Djalon, la graminée pérenne cespiteuse, Paspalum plicatulum, présente les avantages de mieux s'adapter non seulement aux conditions agroclimatiques de la région mais aussi d'avoir une bonne palatabilité pour les ruminants (Ngampongsai et Chanjula, 2009; Tendonkeng et al., 2015). La culture de cette graminée et surtout l'intensification de son utilisation peuvent constituer un palliatif au rétrécissement des parcours dans cette partie de la Guinée dont les systèmes agraires se caractérisent par des conflits fonciers itératifs entre agriculteurs et éleveurs (Mboko et al., 2013) dus à la pratique des cultures en toute saison de la pomme de terre, de l'aubergine etc.
Les principaux facteurs influençant la croissance, le rendement et la valeur nutritive de cette graminée sont, entre autres, le climat, le niveau de fertilité du sol et le mode d'exploitation du fourrage (Tendonkeng et al., 2011a ; Kiema et al., 2012; Klein et al., 2014). P. plicatulum est proposé pour être utilisé avec profit non seulement dans l'amélioration des pâturages naturels mais également en fourrage vert ou conservé (Ngampongsai et Chanjula, 2009). Sans fertilisation, n'importe quelle forme d'exploitation des plantes fourragères conduit à la diminution du stock en azote du sol surtout quand il s'agit des graminées tropicales (Obulbiga et Kaboré-Zoungrana, 2007). Différentes études ont montré que la fertilisation minérale accélère la croissance des plantes, étale la production fourragère dans le temps et entraîne une importante augmentation de la biomasse (Obulbiga et Kaboré-Zoungrana, 2007 ; Tendonkeng et al., 2011b).

Si des travaux ont été menés ailleurs sur la relation entre la fertilisation et le rendement de nombreuses graminées, (Tendonkeng et al., 2011a; Mboko et al., 2013), aucun n'a encore été réalisé sur l'arrière effet de la fertilisation minérale sous forme de NPK (17-17-17) et du stade phénologique sur la croissance et la production de biomasse de $P$. plicatulum au Plateau Central du Fouta-Djalon. D'où l'objectif du présent travail qui visait à déterminer l'effet résiduel de six niveaux de fumure azotée appliquée après une première année de fertilisation et du stade phénologique sur la croissance et le rendement de $P$. plicatulum. 


\section{MATERIEL ET METHODES Zone d'étude}

L'étude a été conduite à la station de recherche agronomique de Bareng au Plateau Central du Fouta-Djalon de mai 2015 à janvier 2016. La station de recherche agronomique de Bareng se situe au Plateau Central du FoutaDjalon entre $12^{\circ} 70^{\prime}$ et $12^{\circ} 04^{\prime}$ longitude Ouest et entre $10^{\circ} 55^{\prime}$ et $11^{\circ} 68^{\prime}$ latitude Nord avec une altitude moyenne de $925 \mathrm{~m}$. La couverture végétale de cette partie du Fouta-Djalon est celle d'une savane herbeuse et boisée par endroit, parsemée de champs de céréales (fonio, maïs, riz, etc.), de pomme de terre et de patate. Les précipitations varient entre 1600 à $2000 \mathrm{~mm}$ par an. Le climat de cette région se caractérise par une saison des pluies s'étendant de mai à novembre, suivi d'une saison sèche froide de décembre à février puis d'une saison sèche chaude de mars à avril. Le mois de mars est le plus chaud et enregistre une température maximum de $30^{\circ} \mathrm{C}$. Les mois de décembre et janvier sont très froids avec un minimum de $7^{\circ} \mathrm{C}$. Le sol de l'essai est de type ferralitique de texture sablo-limoneux acide $(\mathrm{pH}=5)$, à faible teneur en matière organique et en NPK assimilables.

\section{Dispositif expérimental}

Un dispositif factoriel comparant l'effet résiduel de six doses d'engrais sous forme d'engrais composé ternaire, NPK : 17 $17-17(0,50,100,150,200$ et $250 \mathrm{Kg} \mathrm{N} / \mathrm{ha})$ et trois stades phénologiques (montaison, floraison et après grenaison) sur des parcelles élémentaires de $8 \mathrm{~m}^{2}(4 \times 2 \mathrm{~m}$, espacées entre elles de $0,5 \mathrm{~m}$ et $1 \mathrm{~m}$ entre les blocs) en quatre répétitions, soit un total de 72 parcelles expérimentales a été utilisé. Les échantillons du sol $(\mathrm{n}=5)$ ont été prélevés à la deuxième année, sur le site expérimental dans l'horizon $0-20 \mathrm{~cm}$ après la coupe de régularisation des plantes, à l'aide d'une sonde sur les parcelles témoins. L'analyse du sol a été effectuée au Laboratoire d'Analyse des Sols, de Chimie et de l'Environnement (LABASCE) de l'Université de Dschang au Cameroun suivant la méthode décrite par Pauwel et al. (1992) cités par Sawa et al. (2018). D'après le triangle textural de la FAO, la classe texturale moyenne de ce sol est de type sablo-limoneux. C'est également un sol acide $(\mathrm{pH}=5)$ avec une porosité moyenne. Il est pauvre en éléments nutritifs majeurs assimilables $(0,13$ $\mathrm{g} / \mathrm{kg}$ pour l'azote, $0,195 \mathrm{méq} / 100 \mathrm{~g}$ pour le potassium et $2,23 \mathrm{mg} / \mathrm{kg}$ pour le phosphore) et en matière organique $(5,28 \%)$. La valeur du rapport $\mathrm{C} / \mathrm{N}<20$, indique une bonne minéralisation de la matière organique (Tendonkeng et al., 2011a). La teneur en bases échangeables (SBE) a été modérée $(10,88$ méq $/ 100 \mathrm{~g})$. D'après Beernart et Bitondo (1992) cité par Tendonkeng et al. (2011b), la Capacité d'Echange Cationique (CEC) de 9,62 serait qualifiée de faible $(<20$ méq/100 g). Ces observations montrent que ce sol ne peut retenir les ions pour la nutrition des plantes, caractéristiques propres aux oxisols. Une amélioration de la CEC afin que tout engrais répandu y soit retenu pour être mis à la disposition des plantes est donc nécessaire (Tendonkeng et al., 2011b).

\section{Préparation du sol, mise en place des plantes et fertilisation}

Le site de l'expérimentation a été labouré à la première année de l'essai à l'aide d'un tracteur. Le parcellement a été fait manuellement. Au cours de la première année, une quantité de $80 \mathrm{~g}$ d'engrais phosphaté sous la forme de superphosphate $(46 \%$ de phosphore) simple a été appliquée sur chaque parcelle élémentaire comme engrais de fond. En première année, les éclats de souche de Paspalum plicatulum ont été prélevés dans la base fourragère de la Station de Recherche Agronomique de Bareng. Ils ont été habillés (réduction des racines et des feuilles) puis repiqués sur chaque parcelle élémentaire à 4 $\mathrm{cm}$ de profondeur suivant un écartement de 25 $\mathrm{cm} \times 25 \mathrm{~cm}$. Un mois après la plantation des éclats de souche, une coupe de régularisation a été effectuée à $20 \mathrm{~cm}$ au-dessus du sol et les parcelles fertilisées une seule fois aux différentes doses d'engrais. L'effet résiduel de cette fertilisation a été évalué à la seconde année sur les plantes de Paspalum plicatulum. Ainsi, dès le début de la seconde saison pluvieuse de l'essai (début mai), une fauche de régulation des plantes de $P$. plicatulum a été réalisée à la hauteur de $20 \mathrm{~cm}$ au-dessus du sol à l'aide d'une cisaille. Au cours de cette deuxième année de l'essai, aucune autre 
fertilisation n'a été apportée aux plantes de Paspalum plicatulum. L'entretien des parcelles a consisté en l'arrachage manuel des mauvaises herbes et au nettoyage des allées entre les différents blocs et parcelles aux besoins.

\section{Collecte des données}

L'effet résiduel de six niveaux de fertilisation minérale $(0,50,100,150,200$ et $250 \mathrm{~kg} \mathrm{~N} / \mathrm{ha}$ ) sous forme d'engrais composé ternaire NPK (17-17-17) appliquée à l'année précédente et du stade phénologique sur la croissance et le diamètre des plantes de Paspalum plicatulum a été évaluée à chaque stade phénologique sur 16 plantes prélevées au hasard en quatre répétitions pour un total de 64 plantes par traitement. La hauteur des plantes a été mesurée à l'aide d'un mètre ruban gradué au centimètre et le diamètre à l'aide d'un pied à coulisse électronique réglé au millimètre de marque Guanglu LG $^{\circledR}$ (Tendonkeng et al., 2011b). Pendant les périodes de coupe et pour éviter les effets de bordure, les plantes de $P$. plicatulum étaient fauchées à $5 \mathrm{~cm}$ au-dessus du sol au centre de la planche sur une placette utile de $2 \mathrm{~m}^{2}$ (2 x 1 $\mathrm{m})$ bien délimitée puis pesées. A chaque stade phénologique, un échantillon représentatif de la biomasse fauchée par niveau de fertilisation $(500 \mathrm{~g})$ a été prélevé, séparé en feuilles et en tiges et séché dans une étuve ventilée à $60{ }^{\circ} \mathrm{C}$ jusqu'au poids constant pour la détermination de la matière sèche et l'évaluation du rendement. Leur proportion a été exprimée en matière sèche (Obulbiga et KaboréZoungrana, 2007 ; Tendonkeng et al., 2011b ; Mboko et al., 2013).

\section{Analyse statistique}

Les données collectées sur la croissance (hauteur et diamètre) et la biomasse produite ont été soumises à l'analyse de variance suivant le Model Linéaire Général (MLG). Lorsque les différences existaient entre les différents traitements, les moyennes étaient séparées par le test de Duncan au seuil de signification 5\% (Steel et Torrie, 1980 cité par Mboko et al., 2013).

\section{RESULTATS}

Effet résiduel de la fertilisation sur la taille et le diamètre de Paspalum plicatulum à différents stades phénologiques

La hauteur des plantes de $P$. plicatulum a augmenté avec l'effet résiduel des niveaux croissants de fertilisation quel que soit le stade phénologique de la graminée cespiteuse pérenne (Tableau 1). L'effet résiduel de la fertilisation aux doses 200 et $250 \mathrm{~kg} \mathrm{~N} / \mathrm{ha}$ a permis d'obtenir des plantes ayant une hauteur supérieure $(\mathrm{p}<0,05)$ à celle des plantes issues des parcelles non fertilisées et celles produites dans les parcelles ayant reçu respectivement les doses $50 ; 100$ et $150 \mathrm{~kg} \mathrm{~N} / \mathrm{ha}$ à la montaison, à la floraison et après grenaison. Les hauteurs des plantes des parcelles ayant reçu à la première année les doses 200 et 250 $\mathrm{kg} \mathrm{N} /$ ha étaient comparables $(\mathrm{p}>0,05)$ quel que soit le stade phénologique tandis que la taille la plus faible $(\mathrm{p}<0,05)$, a été observée chez les plantes produites sur des parcelles non fertilisées comparativement aux autres doses. A la floraison et après grenaison, les plantes des parcelles fertilisées au cours de l'année précédente, aux doses 100 et $150 \mathrm{~kg}$ $\mathrm{N} / \mathrm{ha}$ avaient les hauteurs comparables ( $\mathrm{p}>$ $0,05)$. D'une manière générale, le niveau croissant de la fertilisation au NPK (17-17-17) a contribué à augmenter la taille des plantes à la deuxième année quel que soit le stade phénologique de $P$. plicatulum.

Le diamètre des plantes de $P$. plicatulum a augmenté avec le niveau croissant de fertilisation quel que soit le stade phénologique (Tableau 2). Ainsi, le diamètre le plus élevé ( $\mathrm{p}<0,05)$ a été obtenu chez les plantes des parcelles ayant reçu au cours de la première année la dose $250 \mathrm{~kg} \quad \mathrm{~N} / \mathrm{ha}$. Cependant, aucune différence significative ( $\mathrm{p}$ $>0,05)$ n'a été observée entre les diamètres des plantes des parcelles ayant reçu les doses 200 et $250 \mathrm{~kg} \mathrm{~N} / \mathrm{ha}$. Par ailleurs, les diamètres des plantes issues des parcelles fertilisées aux doses 200 et $250 \mathrm{~kg}$ N/ha étaient plus élevés $(\mathrm{p}<0,05)$ que ceux des plantes des parcelles non fertilisées et ceux des plantes des parcelles fertilisées aux doses $50 ; 100$ et 150 $\mathrm{kg} \mathrm{N} / \mathrm{ha}$ à la floraison et après grenaison. Par contre, les plantes des parcelles ayant reçu à la première année les doses 150 et $200 \mathrm{~kg} \mathrm{~N} / \mathrm{ha}$ 
avaient des diamètres comparables $(\mathrm{p}>0,05)$ à la montaison. De façon globale, l'effet résiduel de la fertilisation minérale au NPK (17-17-17), a permis d'obtenir des plantes ayant des diamètres supérieurs $(\mathrm{p}<0,05)$ quel que soit le stade phénologique pour les parcelles fertilisées à la dose $250 \mathrm{~kg} \mathrm{~N} / \mathrm{ha}$ suivies de la dose $200 \mathrm{~kg} \mathrm{~N} / \mathrm{ha}$.

\section{Influence du stade phénologique sur la hauteur et le diamètre de Paspalum plicatulum}

La taille de $P$. plicatulum a augmenté $(\mathrm{p}<0,05)$ avec le stade phénologique (Figure 1). La taille des plantes la plus élevée a été observée au stade après grenaison. Par conséquent, aucune différence significative ( $\mathrm{p}$ $>0,05)$ n'a été observée entre la taille des plantes à la floraison et après grenaison.

Le diamètre des plantes a augmenté avec le stade phénologique (Figure 2). Les plantes à la floraison et après grenaison ont présenté les diamètres significativement $(\mathrm{p}<$ $0,05)$ supérieurs à ceux des plantes à la montaison. Aucune différence significative $(\mathrm{P}$ $>0,05)$ n'a été observée entre les diamètres des plantes à la floraison et après grenaison.

\section{Effet résiduel de la fertilisation sur la production de biomasse (t MS/ha) de Paspalum plicatulum à différents stades phénologiques}

La biomasse de la plante entière, des feuilles et des tiges de $P$. plicatulum a augmenté avec le niveau résiduel croissant de la fertilisation (Tableau 3). La plus grande (p $<0,05)$ production de biomasse a été obtenue dans les parcelles ayant reçu à la première année la dose $250 \mathrm{~kg} \mathrm{~N} / \mathrm{ha}$. A la montaison, la biomasse des feuilles a été supérieure $(\mathrm{p}<$ $0,05)$ à celle des tiges, quel que soit le niveau de fertilisation résiduelle. A ce stade, l'effet résiduel résultant de la fertilisation aux doses 200 et $250 \mathrm{~kg} \mathrm{~N} / \mathrm{ha}$ a permis d'obtenir des biomasses supérieures $(\mathrm{p}<0,05)$ à celles obtenues dans les autres parcelles qui, par ailleurs sont restées comparables $(p>0,05)$. Toutes les parcelles fertilisées à la première année, ont présenté des biomasses de la plante entière, des feuilles et des tiges supérieures ( $\mathrm{p}$ $<0,05)$ à celles des parcelles n'ayant pas été fertilisées.
A la floraison, les biomasses des plantes entières, des feuilles et des tiges de $P$. plicatulum ont augmenté en fonction de la dose croissante de fertilisation résiduelle. Par ailleurs, les biomasses des plantes entières, des feuilles et des tiges obtenues à partir des parcelles ayant reçu à la première année les doses 200 et $250 \mathrm{~kg} \mathrm{~N} / \mathrm{ha}$ étaient comparables $(p>0,05)$. A ce stade, la biomasse des tiges a été supérieure à celle des feuilles quel que soit le niveau résiduel de fertilisation. De plus, l'effet résiduel résultant de la fertilisation aux doses 200 et $250 \mathrm{~kg} \mathrm{~N} / \mathrm{ha}$ a permis d'obtenir des biomasses des plantes entières, des feuilles et des tiges supérieures $(p<0,05)$ à celles des autres parcelles.

Au stade après grenaison, la biomasse de la plante entière, des feuilles et des tiges de $P$. plicatulum a augmenté $(\mathrm{p}<0,05)$ pour toutes les parcelles fertilisées comparativement aux parcelles non fertilisées à la première année. A ce stade, l'effet résiduel de la fertilisation à $250 \mathrm{~kg} \mathrm{~N} / \mathrm{ha}$ a permis d'obtenir les biomasses de la plante entière (14,32 t MS/ha), des feuilles (7,00 t MS/ha) et des tiges (7,45 t MS/ha) les plus élevées $(\mathrm{p}<0,05)$. D'une manière générale, l'effet résiduel de la fertilisation au NPK (1717-17) a permis d'accroître les biomasses des tiges, des feuilles et de la plante entière de $P$. plicatulum après grenaison. De même, il a permis également d'obtenir la production de biomasse la plus élevée de la plante entière à la montaison (8,52 t MS/ha).

\section{Effet du stade phénologique sur la production de biomasse de Paspalum plicatulum}

Les biomasses de la plante entière (10,83 t MS/ha), des feuilles (5,07 t MS/ha) et des tiges $(5,76 \mathrm{t} \mathrm{MS} / \mathrm{ha})$ les plus élevées $(\mathrm{p}<$ $0,05)$ ont été obtenues après grenaison (Tableau 4). La plus faible biomasse des tiges a été obtenue à la montaison (2,45 t MS/ha). Aucune différence significative $(\mathrm{p}>0,05) \mathrm{n}$ 'a été observée entre la biomasse de la plante entière, des feuilles et des tiges de $P$. plicatulum à la floraison et après grenaison. Par ailleurs, les biomasses obtenues pour les différentes parties de la plante à la floraison et après grenaison étaient supérieures $(\mathrm{p}<0,05)$ à celles observées à la montaison. 
Tableau 1: Effet résiduel de la fertilisation sur la hauteur $(\mathrm{cm})$ de Paspalum plicatulum à différents stades phénologiques.

\begin{tabular}{cccc}
\hline Fertilisation $(\mathbf{k g ~ N} / \mathbf{h a})$ & Montaison & Floraison & Après grenaison \\
\hline 0 & $44,72^{\mathrm{a}}$ & $64,60^{\mathrm{a}}$ & $67,48^{\mathrm{a}}$ \\
50 & $56,12^{\mathrm{b}}$ & $71,10^{\mathrm{b}}$ & $76,38^{\mathrm{b}}$ \\
100 & $60,54^{\mathrm{c}}$ & $92,72^{\mathrm{c}}$ & $98,26^{\mathrm{c}}$ \\
150 & $66,76^{\mathrm{d}}$ & $92,00^{\mathrm{c}}$ & $98,79^{\mathrm{c}}$ \\
200 & $79,51^{\mathrm{e}}$ & $104,20^{\mathrm{d}}$ & $111,69^{\mathrm{d}}$ \\
250 & $76,44^{\mathrm{e}}$ & $102,17^{\mathrm{d}}$ & $109,35^{\mathrm{d}}$ \\
SEM & 1,022 & 1,035 & 0,909 \\
Prob & 0,00 & 0,00 & 0,00 \\
\hline
\end{tabular}

a,b,c,d,e : les moyennes portant les mêmes lettres dans la même colonne ne sont pas significativement différentes au seuil de 5\%. SEM : Standard Error of the Mean ; Prob: Probabilité.

Tableau 2 : Effet résiduel de la fertilisation sur le diamètre $(\mathrm{cm})$ de Paspalum plicatulum à différents stades phénologiques.

\begin{tabular}{cccc}
\hline Fertilisation (kg N/ha) & Montaison & Floraison & Après grenaison \\
\hline 0 & $0,37^{\mathrm{a}}$ & $0,40^{\mathrm{a}}$ & $0,42^{\mathrm{a}}$ \\
50 & $0,43^{\mathrm{b}}$ & $0,46^{\mathrm{b}}$ & $0,53^{\mathrm{b}}$ \\
100 & $0,48^{\mathrm{c}}$ & $0,56^{\mathrm{c}}$ & $0,61^{\mathrm{c}}$ \\
150 & $0,54^{\mathrm{d}}$ & $0,53^{\mathrm{c}}$ & $0,64^{\mathrm{d}}$ \\
200 & $0,56^{\mathrm{de}}$ & $0,62^{\mathrm{d}}$ & $0,73^{\mathrm{e}}$ \\
250 & $058^{\mathrm{e}}$ & $0,64^{\mathrm{d}}$ & $0,73^{\mathrm{e}}$ \\
SEM & 0,008 & 0,010 & 0,008 \\
Prob & 0,00 & 0,00 & 0,00
\end{tabular}

${ }_{a, b, c, d, e}$ : les moyennes portant les mêmes lettres dans la même colonne ne sont pas significativement différentes au seuil de 5\%. SEM : Standard Error of the Mean ; Prob: Probabilité. 


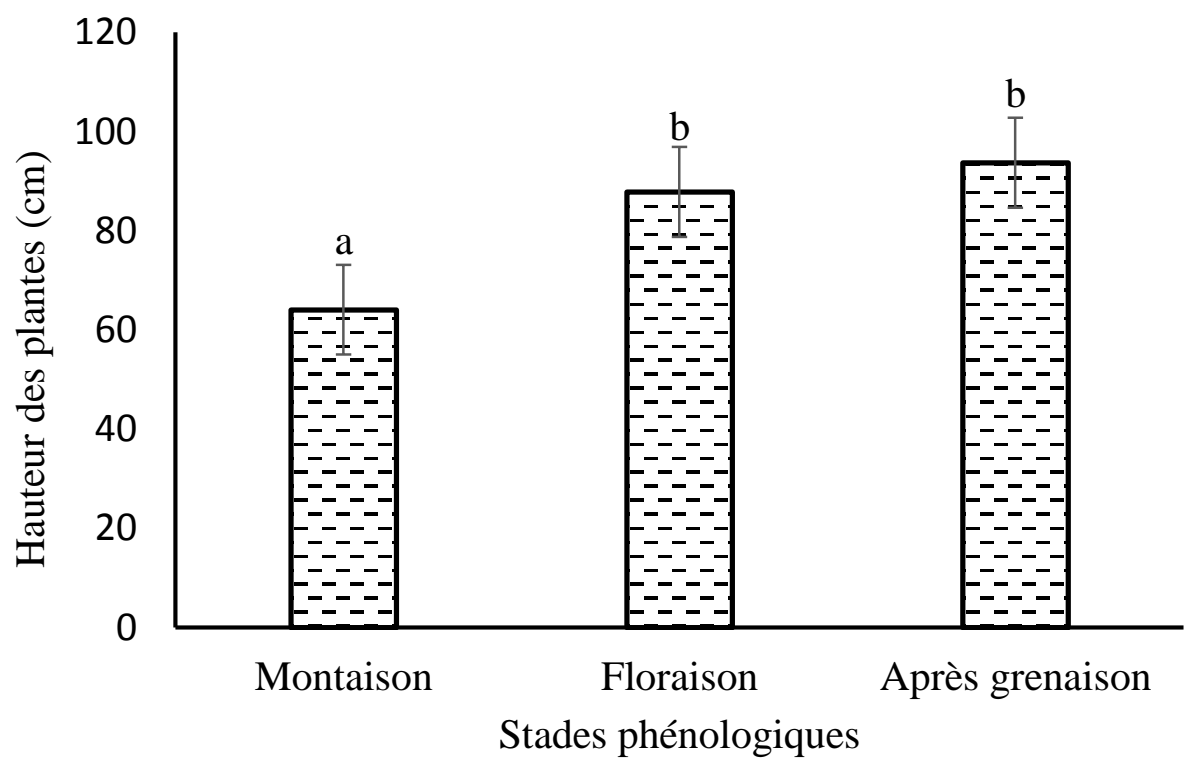

Figure 1 : Evolution de la taille des plantes de P. plicatulum en fonction des stades phénologiques. $a, b:$ les moyennes portant les lettres différentes pour différents stades phénologiques sont significatives au seuil de $5 \%$.

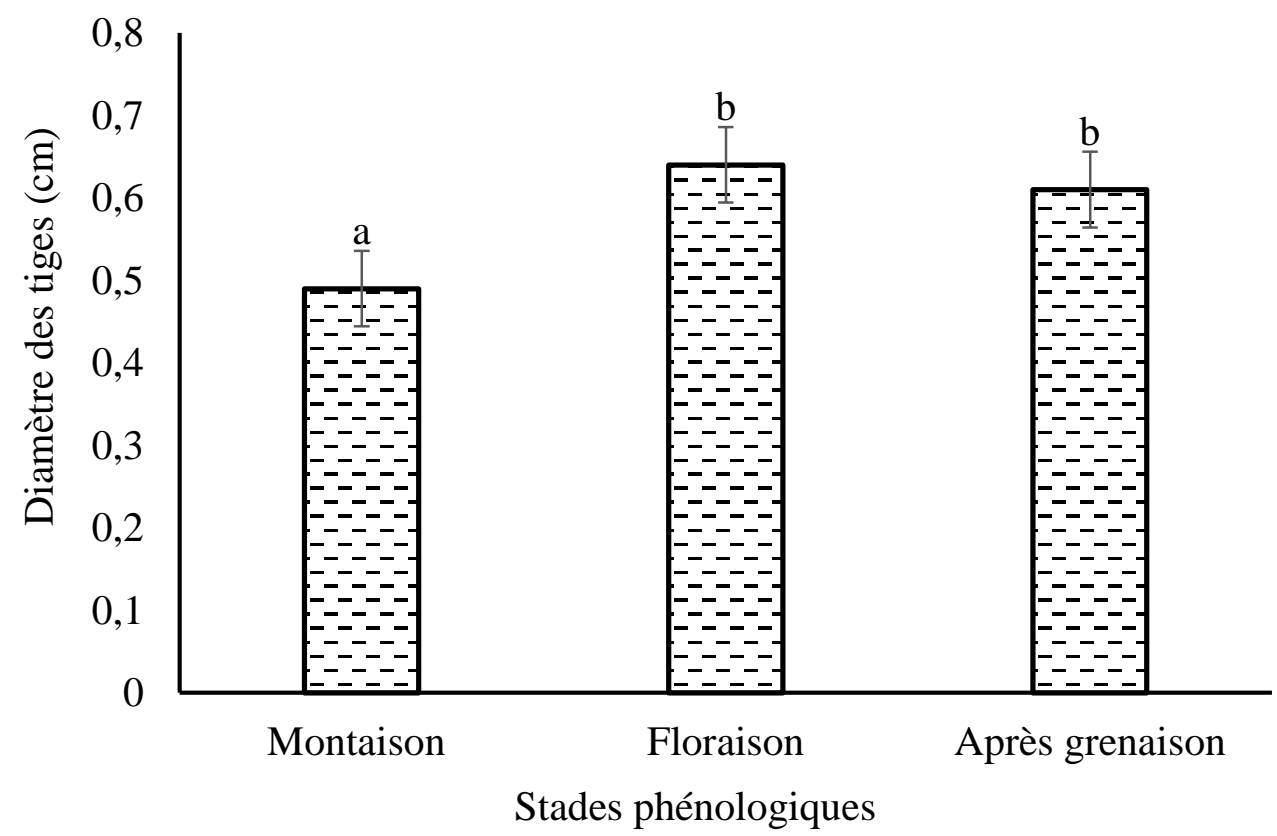

Figure 2: Evolution du diamètre des plantes de $P$. plicatulum en fonction des stades phénologiques.

$a, b$ : les moyennes portant les lettres différentes pour différents stades phénologiques sont significatives au seuil de 5\%. 
C. SAWA et al. / Int. J. Biol. Chem. Sci. 12(5): 2132-2142, 2018

Tableau 3 : Effet résiduel de la fertilisation sur la production de biomasse (t MS/ha) de Paspalum plicatulum à différents stades phénologiques.

\begin{tabular}{|c|c|c|c|c|c|c|c|c|c|}
\hline \multirow{3}{*}{$\begin{array}{l}\text { Fertilisation NPK } \\
\text { (kg N/ha) }\end{array}$} & \multicolumn{9}{|c|}{ Stade phénologiques } \\
\hline & \multicolumn{3}{|c|}{ Montaison } & \multicolumn{3}{|c|}{ Floraison } & \multicolumn{3}{|c|}{ Après grenaison } \\
\hline & Plante entière & Feuilles & Tiges & Plante entière & Feuilles & Tiges & Plante entière & Feuilles & Tiges \\
\hline 0 & $3,30^{\mathrm{a}}$ & $1,93^{\mathrm{a}}$ & $1,35^{\mathrm{a}}$ & $4,44^{\mathrm{a}}$ & $2,00^{\mathrm{a}}$ & $2,45^{\mathrm{a}}$ & $4,53^{\mathrm{a}}$ & $2,00^{\mathrm{a}}$ & $2,53^{\mathrm{a}}$ \\
\hline 50 & $4,10^{\mathrm{b}}$ & $2,40^{\mathrm{b}}$ & $1,70^{\mathrm{b}}$ & $8,43^{\mathrm{b}}$ & $3,96^{\mathrm{b}}$ & $4,50^{\mathrm{b}}$ & $8,91^{b}$ & $4,05^{\mathrm{b}}$ & $4,86^{\mathrm{b}}$ \\
\hline 100 & $6,67^{\mathrm{c}}$ & $3,72^{\mathrm{c}}$ & $2,45^{\mathrm{c}}$ & $10,60^{\mathrm{c}}$ & $5,06 \mathrm{c}$ & $5,53^{\mathrm{c}}$ & $11,15^{\mathrm{c}}$ & $5,05^{\mathrm{c}}$ & $6,10^{\mathrm{c}}$ \\
\hline 150 & $7,30^{\mathrm{d}}$ & $4,43^{\mathrm{d}}$ & $2,86^{\mathrm{d}}$ & $12,00^{\mathrm{d}}$ & $5,60 \mathrm{~d}$ & $6,41^{\mathrm{d}}$ & $12,40^{\mathrm{d}}$ & $6,00^{\mathrm{d}}$ & $6,50^{\mathrm{c}}$ \\
\hline 200 & $8,21^{\mathrm{e}}$ & $5,00^{\mathrm{e}}$ & $3,22^{\mathrm{e}}$ & $13,00^{\mathrm{e}}$ & $6,13^{\mathrm{e}}$ & $7,00 \mathrm{~d}^{\mathrm{e}}$ & $13,70^{\mathrm{e}}$ & $6,60^{\mathrm{e}}$ & $7,12^{\mathrm{d}}$ \\
\hline 250 & $8,52^{\mathrm{e}}$ & $5,40^{\mathrm{e}}$ & $3,15^{\mathrm{e}}$ & $13,46^{\mathrm{e}}$ & $6,40^{\mathrm{e}}$ & $7,10^{\mathrm{e}}$ & $14,32^{\mathrm{e}}$ & $7,00^{\mathrm{e}}$ & $7,45^{\mathrm{d}}$ \\
\hline SEM & 0,243 & 0,136 & 0,112 & 0,233 & 0,121 & 0,128 & 0,323 & 0,163 & 0,165 \\
\hline Prob & 0,00 & 0,00 & 0,00 & 0,00 & 0,00 & 0,00 & 0,00 & 0,00 & 0,00 \\
\hline
\end{tabular}
$a, b, c, d, e$ : les moyennes portant les lettres différentes dans la même colonne sont significatives au seuil de $5 \%$. PE = Plante entière; F = Feuilles; T = Tiges. SEM = Standard Error of the Mean ; Prob $=$
Probabilité. 
Tableau 4 : Biomasse de la plante entière, des tiges et des feuilles de Paspalum plicatulum en fonction des stades phénologiques.

\begin{tabular}{lccc}
\hline Stades phénologiques & \multicolumn{3}{c}{ Biomasse (t MS/ha) } \\
\cline { 2 - 4 } & Plante entière & Feuilles & Tiges \\
\hline Montaison & $6,26^{\mathrm{a}}$ & $3,80^{\mathrm{a}}$ & $2,45^{\mathrm{a}}$ \\
Floraison & $10,30^{\mathrm{b}}$ & $4,84^{\mathrm{b}}$ & $5,45^{\mathrm{b}}$ \\
Après grenaison & $10,83^{\mathrm{b}}$ & $5,07^{\mathrm{b}}$ & $5,76^{\mathrm{b}}$ \\
SEM & 0,855 & 0,314 & 0,295 \\
Prob & 0,00 & 0,00 & 0,00 \\
\hline $\begin{array}{l}a, b \\
\text { Erros } \text { mof the Mean } ; \text { Prob: Probabilité. }\end{array}$ & &
\end{tabular}

\section{DISCUSSION}

La taille et le diamètre des plantes ont augmenté en fonction de l'effet résiduel de différents niveaux de fertilisation. Cette observation corrobore celle formulée par Tendonkeng et al. (2011), sur l'effet résiduel de la fertilisation azotée sous forme d'urée des plantes de Brachiaria ruziziensis en zone d'altitude à l'Ouest du Cameroun similaire au Plateau Central du Fouta-Djalon. L'arrière effet de la fertilisation minérale équivalent azoté sous forme de NPK (17-17-17) a contribué de manière significative $(\mathrm{P}<0,05)$ à l'augmentation de la production de biomasse (t MS/ha) de la plante entière, des feuilles et de des tiges de $P$. plicatulum. Cela est en accord avec les résultats obtenus par de nombreux auteurs (Obulbiga et KaboréZoungrana, 2007; Pamo et al., 2008 ; Tendonkeng et al., 2011). Ceci pourrait s'expliquer par l'évolution du rapport $\mathrm{C} / \mathrm{N}$ pour se retrouver dans la fourchette de l'équilibre idéal pour une bonne minéralisation à la deuxième année après l'application des différentes doses d'engrais composé (NPK: 17-17-17). La bonne minéralisation de l'azote du sol par les microorganismes expliquerait les hauteurs des plantes comparables à la floraison et après grenaison (Tendonkeng et al., 2011), mais aussi à la capacité de Paspalum plicatulum de se développer sur des sols pauvres et acides (Machado, 2013 ; Graminho et al., 2017).

$\mathrm{La}$ variation de la biomasse de Paspalum plicatulum obtenue dans cette étude est proche des résultats obtenus par Graminho et al. (2017) qui ont montré que la biomasse de Paspalum plicatulum. variait de 8 à $19 \mathrm{t}$ MS/ha en fonction du niveau de fertilité du sol et des précipitations. En effet, la fertilisation accroît la vitesse de croissance, ce qui augmente la production pour un stade de développement donné et réduit le délai nécessaire pour atteindre un rendement défini (Tendonkeng et al., 2011).

L'augmentation de la croissance et de la production de biomasse de $P$. plicatulum avec l'évolution des stades phénologiques de la plante observée au cours de cette étude corrobore bien avec les résultats des travaux menés par Tendonkeng et al. (2011) sur la production de Brachiaria ruziziensis à l'Ouest Cameroun. En effet, au cours de la croissance végétale chez les graminées pérennes, il y a augmentation de la densité de talles, le développement de nouveaux bourgeons, des tiges, et l'accélération de l'élongation des feuilles (Tendonkeng et al., 2011). Par ailleurs, les graminées pérennes fauchées au stade jeune, sont pauvres en matière sèche, et ont une teneur en humidité élevée. A la floraison, les nutriments sont mobilisés pour 
la formation des graines et la matière sèche. Ainsi, la proportion des tiges devient plus importante, ce qui a pour conséquence, l'augmentation de la biomasse à la floraison et après grenaison (Tendonkeng et al., 2011).

\section{Conclusion}

Au terme de cette étude, il ressort :

- que la croissance et le rendement des plantes de Paspalum plicatulum ont augmenté $\quad(\mathrm{P}<0,05)$ avec l'effet résiduel des différents niveaux croissants de la fertilisation minérale quel que soit le stade phénologique.

- qu'aucune baisse de la production de biomasse n'a été observée

- que la plus grande biomasse de la plante a été obtenue à la floraison et après grenaison.

- que la floraison est le stade phénologique le mieux indiqué pour une meilleure exploitation de cette graminée fourragère.

\section{CONFLIT D'INTERETS}

Les auteurs de ce présent travail déclarent sur l'honneur qu'ils n'ont aucun conflit d'intérêts.

\section{CONTRIBUTIONS DES AUTEURS}

CS a conçu, a mis en place le dispositif expérimental, a réalisé des essais, a collecté des données et a rédigé le manuscrit; FB et MK ont contribué à la mise en place du dispositif expérimental et à la collecte des données. EM, FT et EPT ont participé à l'assistance technique et scientifique du travail et à la lecture critique du manuscrit. Tous les auteurs ont lu et approuvé la version finale de ce travail.

\section{REMERCIEMENTS}

Nous tenons à adresser toute notre profonde gratitude à la Coordination déléguée du Programme de Productivité Agricole en Afrique de l'Ouest/West Africa Agricultural Productivity Programme (PPAAO/WAAPP 1C AF) de Guinée pour sa contribution financière sans laquelle la présente étude n'aurait pas eu lieu.

\section{REFERENCES}

Fogang Zogang B, Tendonkeng F, Camara Sawa, Boukila B, Pamo ET. 2013. Effets de l'inclusion de différents niveaux de feuilles de Tithonia diversifolia dans des blocs multinutritionnels sur l'ingestion et la digestibilité in vivo de rations à base de paille de Brachiaria ruziziensis chez la brebis Djallonké. Revue d'Elevage et de Médecine Vétérinaire des Pays Tropicaux, 66(3) : 103-107. DOI : 10.19182/remvt.10133.

Graminho LA, Dall'Agnol M, Pötter L, Lopes RR, Simioni C, Weiler Luís R. 2017. Forage characters of different Paspalum species in Rio Grande do Sul: a meta-analysis. Ciência Rural, 47(7). http://dx.doi.org/10.1590/0103$8478 \mathrm{cr} 20161049$.

Kiema A, Nianogo AJ, Kabore-Zoungrana CY, Jalloh B. 2012. Effets des demilunes associées au scarifiage sur les productions fourragères en région sahélienne du Burkina Faso. Int. J. Biol. Chem. Sci., 6(6) : 4018-4030. DOI : http://dx.doi.org/10.4314/ijbcs.v6i6.13.

Klein HD, Rippstein G, Huguenin J, Toutain B, Guerin H, Louppe D. 2014. Les Cultures Fourragères. Edition Quæ, CT, Presses Agronomiques de Gembloux. 262p.

Machado, JM. 2013. Morphogenesis of native grasses of Pampa Biome under nitrogen fertilization. Revista Brasileira de Zootecnia, 42(1): 22-29.

Mboko AV, Tendonkeng F, Matumuini NEF, Zougou TG, Miegoue E, Boukila B, Pamo TE. 2013. Effet comparé de l'enfouissement de deux légumineuses fertilisées au molybdène sur la croissance et le rendement de Brachiaria ruziziensis à différentes périodes de fauche dans l'Ouest Cameroun. Int. J. Biol. Chem. Sci., 7(6): 2513-2525. DOI: 10.4314/ijbcs.v7i6.26.

Ngampongsai W, Chanjula P. 2009. Effect of different levels of sago palm pith on 
nutrient utilization in Thai native cattle fed with Paspalum plicatulum hay (Paspalum plicatulum Michx.) and soybean meal. Songklanakarin J. Sci. Technol., 31(2) : 117-124.

Obulbiga MF, Kaboré-Zoungrana CY. 2007. Influence de la fumure azotée et du rythme d'exploitation sur la production de matière sèche et la valeur alimentaire de Andropogon gayanus Kunth au Burkina Faso. Tropicultura, 25(3) : 161167.

Obulbiga MF, Bougouma V, Sanon HO. 2015. Amélioration de l'offre fourragère par l'association culturale céréalelégumineuse à double usage en zone nord soudanienne du Burkina Faso. Int. J. Biol. Chem. Sci., 9(3): 1431-1439. DOI:

http://dx.doi.org/10.4314/ijbcs.v9i3.26

Pamo TE, Tendonkeng F, Kana JR, Boukila B, Nanda AS. 2006. Effect Calliandra calothyrsus and Leucaena leucocephala supplementary feeding goat production in Cameroon. Small Ruminant Research, 65: 31-37.

Pamo TE, Boukila B, Fonteh FA, Tendonkeng F, Kana JR, Nanda AS. 2007. Nutritive values of some basic grasses and leguminous tree foliage of the Central region of Africa. Animal Feed Science and Technology, 135: 273-282.

Pamo TE, Boukila B, Meduke CN, Tendonkeng F. 2008. Effect of nitrogen fertilisation and cutting frequency on the yield and regrowth of Panicum maximum Jacq in West Cameroon. In: XXI International Grassland Congress / VIII International Rangeland Congress
Hohhot, China, 29th June -5th July 2008, Xie Haining, Huang Jiehua (Eds). p. 354.

Sawa C, Tendonkeng F, Miégoué E, Fogang ZB, Kambale MZ, Kourouma M, Pamo TE. 2018. Effect of different levels of mineral fertilization and phonological stage on growth and Biomass production of Paspalum plicatulum. Journal of Animal Husbandry and Dairy Science, 2(3): 1-8. $\quad$ DOI: $\quad$ http: //dx.doi.org/10.1590/0103

Tendonkeng F, Boukila B, Pamo T.E, Mboko AV, Matumuini NEF. 2011a. Effets direct et résiduel de différents niveaux de fertilisation azotée sur la croissance et le rendement de Brachiaria ruziziensis à différents stades phénologiques. Tropicultura, 29(4) : 197-204.

Tendonkeng F, Boukila B, Pamo TE, Mboko AV, Zogang BF, Matumuini NEF. 2011b. Effets direct et résiduel de différents niveaux de fertilisation azotée sur la composition chimique de Brachiaria ruziziensis à la floraison à l'Ouest Cameroun. Int. J. Biol. Chem. Sci., 5(2) : 570-585. DOI : 10.4314/ijbcs.v5i2.72115.

Tendonkeng F, Mboko AV, Fogang ZB, Matumuini NEF, Miégoué $\mathrm{E}$, Lemoufouet J, Kamo TH, Boukila B. and Pamo TE. 2015. In Vitro Digestibility of Imperata Cylindrica Straw Associated with Multinutrient Block with Inclusion of Different Levels of Tithonia Diversifolia Leaves. J Anim Sci Adv., 5(5) : 1253-1265. DOI: 10.5455/jasa.20150520081023. 